Pacific Journal of Mathematic 


\section{POWER-SERIES AND HAUSDORFF MATRICES}

\section{Philip C. Tonne}

The purpose of this paper is to pair classes of continuous functions from $[0,1]$ to the complex numbers with classes of complex sequences. If $f$ is a function from $[0,1]$ to the complex numbers and $c$ is a complex sequence, a sequence $L(f, c)$ is defined:

$$
L(f, c)_{n}=\sum_{p=0}^{n} f(p / n)\left(\begin{array}{l}
n \\
p
\end{array}\right) \sum_{q=0}^{n-p}(-1)^{q}\left(\begin{array}{c}
n-p \\
q
\end{array}\right) c_{p+q} .
$$

A class $A$ of continuous functions is paired with a class $B$ of sequences provided that

(1) if $f$ is in $A$ and $c$ is in $B$ then $L(f, c)$ converges,

(2) if $f$ is a continuous function and $L(f, c)$ converges for each $c$ in $B$ then $f$ is in $A$, and

(3) if $c$ is a sequence and $L(f, c)$ converges for each $f$ in $A$ then $c$ is in $B$.

We establish the following pairings:

\begin{tabular}{c|c} 
CONTINUOUS & SEQUENCES \\
\hline all continuous functions & Hausdorff moment sequences \\
\hline $\begin{array}{c}\text { power-series absolutely } \\
\text { convergent at } 1\end{array}$ & bounded sequences \\
\hline $\begin{array}{c}\text { power-series absolutely } \\
\text { convergent at } r(r<1)\end{array}$ & $\begin{array}{c}\text { sequences dominated by geo- } \\
\text { metric sequences having ratio } r\end{array}$ \\
\hline entire functions & $\begin{array}{c}\text { all sequences dominated by } \\
\text { geometric sequences }\end{array}$ \\
\hline polynomials & all sequences
\end{tabular}

Felix Hausdorff's work [2] (see also T. H. Hildebrandt [3]) on the moment problem for $[0,1]$ has been continued by J. S. Mac Nerney [5, p. 368 ] to provide the first pairing on the table (see Theorem B). Theorem A, also due to Mac Nerney [6, p. 56], helps establish the last pairing.

THEOREM A. If $f$ is a polynomial and is $c$ is a complex sequence, then the sequence $L(f, c)$ converges. Furthermore, if $f=$ $\sum_{p=0}^{n} A_{p} I^{p}$, where $I$ is the identity function on the complex plane, then $L(f, c)$ has limit $\sum_{p=0}^{n} A_{p} c_{p}$.

THeOREM B. Suppose that $c$ is a complex sequence. Then these are equivalent:

(1) There is a function $g$ of bounded variation from $[0,1]$ to the complex numbers such that, for each non-negative integer $n, c_{n}=\int_{0}^{1} I^{n} d g$. 
(2) For each $f$ in $C[0,1]$, the class of continuous functions from $[0,1]$ to the complex numbers, $L(f, c)$ converges.

Furthermore, if (1) holds and $f$ is in $C[0,1]$, then $L(f, c)$ has $\operatorname{limit} \int_{0}^{1} f d g$.

DEFINITION. If each of $p$ and $n$ is a nonnegative integer and $c$ is a complex sequence, then $\Delta^{0} c_{p}=c_{p}$ and $\Delta^{n+1} c_{p}=\Delta^{n} c_{p}-\Delta^{n} c_{p+1}$.

The following notes are helpful.

Note 1. If each of $m$ and $p$ is a nonnegative integer and $c$ is a complex sequence,

$$
\Delta^{m} c_{p}=\sum_{q=0}^{m}(-1)^{q}\left(\begin{array}{c}
m \\
q
\end{array}\right) c_{p+q},
$$

so that if $f$ is a function from $[0,1]$ to the complex numbers then

$$
L(f, c)_{n}=\sum_{p=0}^{n}\left(\begin{array}{l}
n \\
p
\end{array}\right) \Delta^{n-p} c_{p} f(p / n) .
$$

Definition. If each of $p$ and $k$ is a nonnegative integer, $Y_{p k}=$ $\sum_{q=0}^{p}(-1)^{p+q}\left(\begin{array}{l}p \\ q\end{array}\right) q^{k}$, where we interpret $0^{\circ}$ as 1 .

Note 2.

$$
Y_{p+1, k+1}=(p+1)\left(Y_{p k}+Y_{p+1, k}\right) ; \quad Y_{p p}=p ! ; \quad Y_{p k} \geqq 0 ; \quad Y_{p k}=0
$$

for $p>k$.

Note 3. If $f$ is a function from $[0,1]$ to the complex numbers and $c$ is a complex sequence and $n$ is a positive integer, then

$$
\begin{aligned}
L(f, c)_{n} & =\sum_{p=0}^{n}\left(\begin{array}{l}
n \\
p
\end{array}\right) \sum_{q=0}^{n-p}(-1)^{q}\left(\begin{array}{c}
n-p \\
q
\end{array}\right) c_{p+q} f(p / n) \\
& =\sum_{p=0}^{n} c_{p}\left(\begin{array}{l}
n \\
p
\end{array}\right) \sum_{q=0}^{p}(-1)^{p+q}\left(\begin{array}{l}
p \\
q
\end{array}\right) f(q / n),
\end{aligned}
$$

and, in case there is a complex sequence $A$ such that, for each number $x$ in $[0,1], f(x)=\sum_{k=0}^{\infty} A_{k} x^{k}$, then

$$
\begin{aligned}
L(f, c)_{n} & =\sum_{p=0}^{n} c_{p}\left(\begin{array}{l}
n \\
p
\end{array}\right) \sum_{k=0}^{\infty} A_{k} n^{-k} \sum_{q=0}^{p}(-1)^{p+q}\left(\begin{array}{l}
p \\
q
\end{array}\right) q^{k} \\
& =\sum_{p=0}^{n} c_{p}\left(\begin{array}{l}
n \\
p
\end{array}\right) \sum_{k=p}^{\infty} A_{k} n^{-k} Y_{p k} .
\end{aligned}
$$

The following theorem is useful in a later argument and is stated here for purposes of introduction. 
THEOREM 0. Let $p$ be a nonnegative integer and let $z$ be the sequence whose value at $p$ is 1 and whose value elsewhere is 0 . Let $k$ be a function from $[0,1]$ to the complex numbers which is continuous at 0 . Then $L\left(k \cdot I^{p}, z\right)$ has limit $k(0)$.

Indication of proof. If $n$ is an integer greater than $p$, then

$$
\begin{aligned}
L\left(k \cdot I^{p}, z\right)_{n} & =\left(\begin{array}{c}
n \\
p
\end{array}\right) \sum_{q=0}^{p}(-1)^{p+q}\left(\begin{array}{c}
p \\
q
\end{array}\right) k(q / n) q^{p} n^{-p} \\
& =\left(\begin{array}{c}
n \\
p
\end{array}\right) n^{-p} \sum_{q=0}^{p}(-1)^{p+q}\left(\begin{array}{c}
p \\
q
\end{array}\right) q^{p} k(q / n) ; \\
\lim _{n \rightarrow \infty}\left(\begin{array}{c}
n \\
p
\end{array}\right) n^{-p} & =1 / p ! ; p !=Y_{p p}=\sum_{q=0}^{p}(-1)^{p+q}\left(\begin{array}{c}
p \\
q
\end{array}\right) q^{p} ;
\end{aligned}
$$

and

$$
\begin{aligned}
& \left|\sum_{q=0}^{p}(-1)^{p+q}\left(\begin{array}{c}
p \\
q
\end{array}\right) q^{p} k(q / n)-p ! k(0)\right| \\
& \leqq \sum_{q=0}^{p}\left(\begin{array}{c}
p \\
q
\end{array}\right) q^{p}|k(q / n)-k(0)| .
\end{aligned}
$$

1. Radius of Convergence $\geqq 1$. In this chapter, for each $r$ not less than 1, we pair functions having power-series expansions about 0 which are absolutely convergent at $r$ with sequences which are dominated by geometric sequences with ratio $r$. In particular we match functions with power-series expansions about 0 which are absolutely convergent at 1 with the class of bounded sequences.

Theorem 1. Suppose that $r \geqq 1$, each of $A$ and $c$ is a complex sequence, $\sum_{p=0}^{\infty}\left|A_{p}\right| r^{p}$ converges, there is a number $t$ such that if $p$ is a nonnegative integer then $\left|c_{p}\right| \leqq t \cdot r^{p}$, and $f=\sum_{p=0}^{\infty} A_{p} I^{p}$. Then $L(f, c)$ converges to $\sum_{p=0}^{\infty} A_{p} c_{p}$.

Lemma 1. If $g$ is a function from $[0,1]$ to the complex numbers and $u$ is a constant sequence, then, for each positive integer $n$, $L(g, u)_{n}=u_{0} \cdot g(1)$.

Proof.

$$
L(g, u)_{n}=\sum_{p=0}^{n} g(p / n)\left(\begin{array}{l}
n \\
p
\end{array}\right) \Delta^{n-p} u_{p}=g(n / n)\left(\begin{array}{l}
n \\
n
\end{array}\right) u_{n} .
$$

Lemma 2. Suppose that $d>0$ and $b$ is a complex sequence such that $\sum_{p=0}^{\infty}\left|b_{p}\right|$ converges. Then there is a positive integer $N$ such that if $n$ is an integer greater than $N$ then

$$
\left|\sum_{p=0}^{n} b_{p}\left[1-\left(\begin{array}{l}
n \\
p
\end{array}\right) n^{-p} p !\right]\right|<d .
$$


Proof. We note that if $p$ is a nonnegative integer and $s$ is a sequence such that, for each positive integer $n, s_{n}=\left(\begin{array}{c}n \\ p\end{array}\right) n^{-p} p$ !, then $s$ is nondecreasing with limit 1 .

Let $m$ be a positive integer such that $\sum_{p=m}^{\infty}\left|b_{p}\right|<d / 2$. There is an integer $N$ greater than $m$ such that if $k$ is an integer in $[0, m]$ then, for each integer $n$ greater than $N$,

$$
1-\left(\begin{array}{c}
n \\
k
\end{array}\right) n^{-k} k !<d /\left[2(m+1)\left(\left|b_{k}\right|+1\right)\right] .
$$

If $n$ is an integer greater than $N$,

$$
\begin{aligned}
& \left|\sum_{p=0}^{n} b_{p}\left[1-\left(\begin{array}{c}
n \\
p
\end{array}\right) n^{-p} p !\right]\right| \\
& \quad \leqq \sum_{p=0}^{m}\left|b_{p}\right|\left[1-\left(\begin{array}{c}
n \\
p
\end{array}\right) n^{-p} p !\right]+\sum_{p=m}^{n}\left|b_{p}\right|<d .
\end{aligned}
$$

Lemma 3. Let $b$ be a positive number. Then there is a positive integer $N$ such that if $n$ is an integer greater than $N$ then

$$
\sum_{p=0}^{n}\left(\begin{array}{c}
n \\
p
\end{array}\right) \sum_{k=p+1}^{\infty}\left|A_{k}\right| r^{k} n^{-k} Y_{p k}<b .
$$

Proof. There is a positive integer $m$ such that $\sum_{p=m}^{\infty}\left|A_{p}\right| r^{p}<b / 2$. Let $g$ be $\sum_{p=0}^{\infty}\left|A_{p}\right| r^{p} I^{p}$. Let $N$ be an integer greater than $m$ such that if $n$ is an integer greater than $N$ then

$$
\sum_{p=0}^{n}\left|A_{p}\right| r^{p}\left[1-\left(\begin{array}{c}
n \\
p
\end{array}\right) n^{-p} p !\right]<b / 2 .
$$

Then, if $n$ is an integer greater than $N$,

$$
\begin{aligned}
b / 2> & g(1)-\sum_{p=0}^{n}\left|A_{p}\right| r^{p} \\
= & L(g, 1)_{n}-\sum_{p=0}^{n}\left|A_{p}\right| r^{p} \\
= & \sum_{p=0}^{n}\left[\left(\begin{array}{c}
n \\
p
\end{array}\right) \sum_{k=p}^{\infty}\left|A_{k}\right| r^{k} n^{-k} Y_{p k}-\left|A_{p}\right| r^{p}\right] \\
= & \sum_{p=0}^{n}\left[\left(\begin{array}{c}
n \\
p
\end{array}\right) n^{-p} p !-1\right]\left|A_{p}\right| r^{p} \\
& \quad+\sum_{p=0}^{n}\left(\begin{array}{c}
n \\
p
\end{array}\right) \sum_{k=p+1}^{\infty}\left|A_{k}\right| r^{k} n^{-k} Y_{p k} .
\end{aligned}
$$

so

$$
\begin{aligned}
\sum_{p=0}^{n}\left(\begin{array}{l}
n \\
p
\end{array}\right) \sum_{k=p+1}^{\infty}\left|A_{k}\right| r^{k} n^{-k} Y_{p k} \\
\quad \leqq b / 2+\sum_{p=0}^{n}\left|A_{p}\right| r^{p}\left[1-\left(\begin{array}{c}
n \\
p
\end{array}\right) n^{-p} p !\right]<b .
\end{aligned}
$$


Proof of Theorem 1. Let $\varepsilon$ be a positive number. There is a positive integer $N$ such that if $n$ is an integer greater than $N$ then

$$
\begin{gathered}
\left|\sum_{p=0}^{\infty} A_{p} c_{p}-\sum_{p=0}^{n} A_{p} c_{p}\right|<\epsilon / 2, \\
\sum_{p=0}^{n}\left(\begin{array}{c}
n \\
p
\end{array}\right) \sum_{k=p+1}^{\infty}\left|A_{k}\right| r^{k} n^{-k} Y_{p k}<\varepsilon /(4 t),
\end{gathered}
$$

and

$$
\left|\sum_{p=0}^{n} A_{p} c_{p}\left[1-\left(\begin{array}{c}
n \\
p
\end{array}\right) n^{-p} p !\right]\right|<\varepsilon / 4 .
$$

Hence, if $n$ is an integer greater than $N$,

$$
\begin{gathered}
\left|L(f, c)_{n}-\sum_{p=0}^{\infty} A_{p} c_{p}\right| \\
<\varepsilon / 2+\left|\sum_{p=0}^{n}\left[c_{p}\left(\begin{array}{c}
n \\
p
\end{array}\right) \sum_{k=p}^{\infty} A_{k} n^{-k} Y_{p k}-A_{p} c_{p}\right]\right| \\
\leqq \varepsilon / 2+\left|\sum_{p=0}^{n} c_{p} A_{p}\left[1-\left(\begin{array}{c}
n \\
p
\end{array}\right) n^{-p} p !\right]\right| \\
+t \cdot \sum_{p=0}^{n}\left(\begin{array}{c}
n \\
p
\end{array}\right) \sum_{k=p+1}^{\infty}\left|A_{k}\right| r^{k} n^{-k} Y_{p k} \\
<\varepsilon .
\end{gathered}
$$

Theorem 2. Suppose $r \geqq 1$ and $S$ is the set to which $f$ belongs only if there is a complex sequence $A$ such that $\sum_{p=0}^{\infty}\left|A_{p}\right| r^{p}$ converges and $f=\sum_{p=0}^{\infty} A_{p} I^{p}$. Suppose that $c$ is an infinite complex sequence and, for each $f$ is $S, L(f, c)$ converges. Then $c$ is bounded by a geometric sequence with ratio $r$.

Proof. For each nonnegative integer $p$ let $g_{p}$ be $r^{-p}$, and suppose that the sequence $c \cdot g$ is not bounded; that is, suppose that $c$ is not bounded by a geometric sequence with ratio $r$.

For each $f$ in $S$, let $N(f)$ be $\sum_{p=0}^{\infty} r^{p}\left|f^{(p)}(0)\right| / p$ !. Then $(S, N)$ is a complete, normed, linear space.

For each positive integer $n$, let $T_{n}$ be a function from $S$ to the complex numbers such that if $f$ is in $S$ then $T_{n}(f)=L(f, c)_{n}$. If $f$ is in $S$ and $n$ is a positive integer and $|f|_{[0,1]}$ denotes the maximum modulus of $f$ on $[0,1]$, then

$$
\begin{aligned}
\left|T_{n}(f)\right| & =\left|\sum_{p=0}^{n} c_{p}\left(\begin{array}{l}
n \\
p
\end{array}\right) \sum_{q=0}^{p}(-1)^{p+q}\left(\begin{array}{l}
p \\
q
\end{array}\right) f(q / n)\right| \\
& \leqq|f|_{[0,1]} \sum_{p=0}^{n}\left|c_{p}\right|\left(\begin{array}{l}
n \\
p
\end{array}\right) \sum_{q=0}^{p}\left(\begin{array}{c}
p \\
q
\end{array}\right) \\
& \leqq N(f) \sum_{p=0}^{n}\left|c_{p}\right|\left(\begin{array}{l}
n \\
p
\end{array}\right) 2^{p}
\end{aligned}
$$


so that $T_{n}$ is a continuous linear transformation from $(S, N)$ to the complex numbers.

Let $m$ be a positive integer. $N\left(r^{-m} I^{m}\right)=1$. By Theorem A, $L\left(r^{-m} I^{m}, c\right)$ has limit $g_{m} c_{m}$. Hence, there is a positive integer $n$ such that $\left|T_{n}\left(r^{-m} I^{m}\right)\right|>\left|g_{m} c_{m}\right|-1$, so that the sequence $N^{\prime}[T]$-where, for each positive integer $n, N^{\prime}\left(T_{n}\right)$ is the least number $b$ such that if $F$ is in $S$ and $N(F) \leqq 1$ then $\left|T_{n}(F)\right| \leqq b$-is not bounded. So, by the "principle of uniform boundedness," there is a member $f$ of $S$ such that the sequence $T(f)$ is not bounded, but $L(f, c)$ converges and $T(f)=L(f, \dot{c})$, so the theorem is proved.

THEOREM 3. Suppose that $r>0, f$ is in $C[0,1]$, and, for each complex sequence $c$ which is dominated by a geometric sequence with ratio $r, L(f, c)$ converges. Then there is a complex sequence $A$ such that $\sum_{p=0}^{\infty}\left|A_{p}\right| r^{p}$ converges and, if $x$ is in $[0,1]$ and $x \leqq r$, then $f(x)=\sum_{p=0}^{\infty} A_{p} x^{p}$.

Proof. For each nonnegative-integer pair $(n, p)$, let $g_{n}$ be $r^{n}$ and let $M_{n p}$ be $\left(\begin{array}{c}n \\ p\end{array}\right) \sum_{q=0}^{p}(-1)^{p+q}\left(\begin{array}{l}p \\ q\end{array}\right) f(q / n)$. Then, for each bounded complex sequence $c$ and each positive integer $n, L(f, c \cdot g)_{n}=\sum_{p=0}^{n} c_{n} M_{n p} r^{p}$, so that, by the "principle of uniform boundedness", there is a number $D$ such that, for each positive integer $n, \sum_{p=0}^{n}\left|M_{n p}\right| r^{p}<D$.

For each nonnegative integer $p$, let $z(p)$ be the sequence whose value at $p$ is 1 and whose value elsewhere is 0 . Then the sequence $M[, p]=L(f, z(p))$, which, by hypothesis, has limit, say $A_{p}$, and from the preceding paragraph we see that $\sum_{p=0}^{\infty}\left|A_{p}\right| r^{p}$ converges.

For each positive integer $n$ let $B_{n}(f)$ be the Bernstein polynomial for $f$ of order $n$; i.e., let $B_{n}(f)$ be $\sum_{p=0}^{n} M_{n p} I^{p} . \quad B(f)$ converges to $f$ on $[0,1]$ [see 1 , pp. 1-2; also 4, pp. 5-7]. Now, if $x$ is a complex number and $|x| \leqq r$, then

$$
\left|B_{n}(f)(x)\right| \leqq \sum_{p=0}^{n}|x|^{p}\left|M_{n p}\right|<D .
$$

By the convergence of the Bernstein polynomials on $[0,1]$ and the convergence continuation theorem, a subsequence of $B(f)$ has limit, say $h$, on $[0,1]$ and the disc with center 0 and radius $r . h$ is analytic at 0 . By Theorems 0 and $\mathrm{A}$ we see that if $p$ is a non-negative integer then

$$
\begin{aligned}
h^{(p)}(0) / p ! & =\lim L(h, z(p)) \\
& =\lim L(f, z(p))=A_{p},
\end{aligned}
$$

and, if $x$ is in $[0,1]$ and $x \leqq r$, then $f(x)=h(x)=\sum_{p=0}^{\infty} A_{p} x^{p}$.

The following theorem parts somewhat from the main stream of our study but sheds additional light on the problem at hand. 
THeorem 4. Suppose that $A$ is a complex sequence, $\sum_{p=0}^{\infty}\left|A_{p}\right|$ converges, and $f=\sum_{p=0}^{\infty} A_{p} I^{p}$. Then there is an unbounded numbersequence $c$ such that $L(f, c)$ converges.

Proof. For each positive integer $n$, let $s_{n}$ be

$$
\sum_{p=0}^{n}\left(\begin{array}{c}
n \\
p
\end{array}\right) \sum_{k=p+1}^{\infty}\left|A_{k}\right| n^{-k} Y_{p k}
$$

By Lemma 3, $s$ has limit $0 . A$ has limit 0 . So there is an increasing, nonnegative-integer sequence $u$ such that, for each nonnegativeinteger pair $(p, q), s\left(u_{p}+q\right)<4^{-p}$ and $\left|A\left(u_{p}+q\right)\right|<4^{-p}$.

For each nonnegative integer $p$, let $c_{p}$ be $2^{m}$ if $m$ is a nonnegative integer such that $p=u_{m}$, otherwise let $c_{p}$ be 0 .

If $k$ is a nonnegative integer and $n=u_{k},\left|c_{n} A_{n}\right|<2^{-k}$, so that $\sum_{p=0}^{\infty}\left|A_{p} c_{p}\right|$ converges.

Let $b$ be a positive number. By Lemma 2, there is a positive integer $N$ such that $2^{-N}<b / 2$ and if $n$ is an integer greater than $N$ then

$$
\sum_{p=0}^{n}\left|A_{p} c_{p}\right|\left[1-\left(\begin{array}{c}
n \\
p
\end{array}\right) n^{-p} p !\right]<b / 4
$$

and

$$
\left|\sum_{p=n+1}^{\infty} A_{p} c_{p}\right|<b / 4
$$

Let $n$ be an integer not less than $u_{N}$ and let $m$ be the greatest integer $k$ such that $u_{k} \leqq n$. Then

$$
\begin{gathered}
\left|\sum_{p=0}^{\infty} A_{p} c_{p}-L(f, c)_{n}\right| \\
<b / 4+\left|\sum_{p=0}^{n} A_{p} c_{p}-\sum_{p=0}^{n} c_{p}\left(\begin{array}{c}
n \\
p
\end{array}\right) \sum_{k=p}^{\infty} A_{k} n^{-k} Y_{p k}\right| \\
\leqq b / 4+\sum_{p=0}^{n}\left|A_{p} c_{p}\right|\left[1-\left(\begin{array}{c}
n \\
p
\end{array}\right) n^{-p} p !\right] \\
+\sum_{p=0}^{n} c_{p}\left(\begin{array}{l}
n \\
p
\end{array}\right) \sum_{k=p+1}^{\infty}\left|A_{k}\right| n^{-k} Y_{p k} \\
<b / 2+\sum_{p=0}^{n} 2^{m}\left(\begin{array}{c}
n \\
p
\end{array}\right) \sum_{k=p+1}^{\infty}\left|A_{k}\right| n^{-k} Y_{p k} \\
<b / 2+2^{m} s_{n}<b / 2+2^{m} \cdot 4^{-m}<b,
\end{gathered}
$$

so $L(f, c)$ converges to $\sum_{p=0}^{\infty} A_{p} c_{p}$.

2. Entire functions. Following from Theorems 1 and 3 we have: 
Theorem 5. Suppose that $f$ is in $C[0,1]$. Then the following statements are equivalent:

(1) $f$ is a subset of an entire function.

(2) If $c$ is a complex sequence which is dominated by a geometric sequence, then $L(f, c)$ converges.

Furthermore, if (2) holds, $L(f, c)$ converges to $\sum_{p=0}^{\infty}\left(f^{(p)}(0) / p !\right) c_{p}$.

THeOREm 6. Suppose that $c$ is a complex sequence such that $L(f, c)$ converges for each entire function $f$. Then $c$ is dominated by a geometric sequence.

Proof. Suppose that $c$ is not dominated by a geometric sequence.

LEMmA. If each of $m$ and $r$ is a nonnegative integer, then there is a positive integer $q$ such that $\left|c_{m+q}\right|>r^{m+q+1}$ and $\left|c_{m+q}\right|>2^{m}\left|c_{p}\right|$ for each nonnegative integer $p$ less than $m+q$.

Proof of lemma. Let $R$ be $r+2^{m}+\sum_{p=0}^{m}\left|c_{p}\right|$. Since no geometric sequence dominates $c$, there is a positive integer $k$ such that $\left|c_{m+k}\right|>R^{m+k+1}$. Let $q$ be the least positive integer $n$ such that $\left|c_{m+n}\right|>R^{m+n+1}$.

Suppose that $p$ is a nonnegative integer.

If $p \leqq m$, then $\left|c_{m+q}\right|>R^{m+q+1} \geqq R^{2}>2^{m}\left|c_{p}\right|$.

If $m<p<m+q$, then

$$
\left|c_{m+q}\right|>R^{m+q+1} \geqq R \cdot R^{p+1} \geqq R \cdot\left|c_{p}\right|>2^{m}\left|c_{p}\right| \text {. }
$$

Continuation of proof of Theorem 6. By the lemma, there is an increasing interger-valued sequence $u$ such that $u_{0}=0$ and, if $p$ is a positive integer, then $\left|c\left(u_{p}\right)\right|>p^{u(p)+1}$ and $\left|c\left(u_{p}\right)\right| \geqq 2^{u(p-1)}\left|c_{n}\right|$ for each nonnegative integer $n$ less than $u_{p}$.

Let $f$ be $\sum_{p=1}^{\infty}\left(1 / c\left(u_{p}\right)\right) I^{u(p)}$.

If $N$ is a positive integer then, for each integer $p$ greater than $N$,

$$
\left|\frac{1}{c\left(u_{p}\right)}\right|^{1 / u_{p}}<p^{-(u(p)+1) /(u(p))}<p^{-1}<1 / N,
$$

so that $f$ is an entire function.

For each nonnegative integer $k$ let $A_{k}$ be $f^{(k)}(0) / k$ !. Now, if $p$ and $k$ are integers and $0 \leqq p<k$, then $\left|c_{p} A_{k}\right|<2^{-k}$.

Suppose that $B>0$. By Lemma 3 and the note at the beginning of the proof of Lemma 2 , there is a positive-integer pair $(n, m)$ such that

$$
\begin{gathered}
\sum_{p=0}^{n}\left(\begin{array}{c}
n \\
p
\end{array}\right) \sum_{k=p+1}^{\infty} 2^{-k} n^{-k} Y_{p k}<1, \\
\sum_{p=0}^{m}\left(\begin{array}{c}
n \\
u_{p}
\end{array}\right) n^{-u(p)}\left(u_{p}\right) !>B,
\end{gathered}
$$


and $n \geqq u_{u}$. Then $\left|L(f, c)_{n}\right|$

$$
\begin{gathered}
-\left|\sum_{p=0}^{n} c_{p}\left(\begin{array}{l}
n \\
p
\end{array}\right) \sum_{k=p}^{\infty} A_{k} n^{-k} Y_{p l k}\right| \\
\geqq \sum_{p=0}^{n} c_{p}\left(\begin{array}{l}
n \\
p
\end{array}\right) A_{p} p^{-p} p !-\sum_{p=0}^{n}\left(\begin{array}{c}
n \\
p
\end{array}\right) \sum_{h=p+1}^{\infty}\left|c_{p} A_{k}\right| n^{-k} Y_{p k} \\
\geqq \sum_{p=0}^{m} c\left(u_{p}\right)\left(\begin{array}{l}
n \\
u_{p}
\end{array}\right) A\left(u_{p}\right) n^{-u(p)}\left(u_{p}\right) ! \\
-\sum_{p=0}^{n}\left(\begin{array}{c}
n \\
p
\end{array}\right) \sum_{k=p+1}^{\infty} 2^{-k} n^{-k} Y_{p k}
\end{gathered}
$$

$>B-1$, so that $L(f, c)$ does not converge. Hence, $c$ is dominated by a geometric sequence.

3. A converse to 'Theorem A. The following theorem, together with Theorem A, shows that the last pair on our table belongs there.

Theorem 7. Suppose that $f$ is in $C[0,1]$ and, for each complex sequence $c, L(f, c)$ converges. Then $f$ is a subset of a polynomial.

Proof. By Theorem 3 there is a complex sequence $A$ such that if $x$ is in $[0,1]$ then $f(x)=\sum_{p=0}^{\infty} A_{p} x^{p}$.

For each nonnegative-integer pair $(n, p)$, let $M_{n p}$ be

$$
\left(\begin{array}{l}
n \\
p
\end{array}\right) \sum_{q=0}^{p}(-1)^{p+q}\left(\begin{array}{l}
p \\
q
\end{array}\right) f(q / n),
$$

let $w_{p}$ be $1 / A_{p}$ if $A_{p} \neq 0$ and $w_{p}$ be 0 if $A_{p}=0$, and let $Q_{n p}$ be $w_{p} M_{n p}$. Now, if $x$ is an infinite complex sequence and $T(x)_{n}=\sum_{p=0}^{n} Q_{n p} x_{p}$ for each positive integer $n$, then $T(x)=L(f, w \cdot x)$, so that $T(x)$ converges. Therefore, by the "principle of uniform boundedness," there is a number $B$ such that, for each positive integer $n, \sum_{p=0}^{n}\left|Q_{n p}\right|<B$. Now, if $p$ is a nonnegative integer such that $A_{p} \neq 0$, the sequence $Q[, p]$ has limit 1. Hence, there is a positive integer $N$ such that if $p$ is an integer greater than $N$ then $A_{p}=0$, so $f$ is a subset of a polynomial.

4. Radius of convergence less than 1 . Lemma 1 tells us that constant sequences prevent us from altering Theorem 2 to allow $r$ to be less than 1 .

Theorem 3, as it is, not restricted in this way.

This leaves the question: Can we find anything like Theorem 1 with the radius of convergence for our power-series expansions about 0 less than 1 ?

THEOREM 8. Suppose lhat $0<r<1, f$ is a finction analytic on the disc with center 1 and radius $1+r, \sum_{p=0}^{\infty}\left(\left|f^{(p)}(1)\right| / p !\right)(1+r)^{p}$ 
converges, $c$ is a complex sequence, $t>0$, and, for each nonnegative integer $n,\left|c_{n}\right| \leqq t \cdot r^{n}$. Then $L(f, c)$ converges to $\sum_{p=0}^{\infty}\left(f^{(p)}(0) / p !\right) c_{p}$.

Indication of proof. For each nonnegative integer $n$ let $B_{n}$ be $f^{(n)}(1) / n$ ! and let $d_{n}$ be $\Delta^{n} c_{0}$. Then

$$
\left|d_{n}\right|=\left|\sum_{q=0}^{n}(-1)_{q}\left(\begin{array}{c}
n \\
q
\end{array}\right) c^{q}\right| \leqq t \cdot \sum_{q=0}^{n}\left(\begin{array}{c}
n \\
q
\end{array}\right) r^{q}=t \cdot(1+r)^{n}
$$

For each complex number $z$ such that $|z|<1+r$. let $g(z)$ be $f(1-z)$. Then for each positive integer $n$,

$$
\begin{aligned}
L(f, c)_{n} & =\sum_{p=0}^{n} f(p / n)\left(\begin{array}{l}
n \\
p
\end{array}\right) \Delta^{n-p} c_{p} \\
& =\sum_{p=0}^{n} f(1-p / n)\left(\begin{array}{c}
n \\
n-p
\end{array}\right) \Delta^{p} c_{n-p} \\
& =\sum_{p=0}^{n} g(p / n)\left(\begin{array}{l}
n \\
p
\end{array}\right) \Delta^{n-p} d_{p} \\
& =L(g, d)_{n},
\end{aligned}
$$

so that, by Theorem $1, L(f, c)=L(g, d)$ converges to

$$
\begin{aligned}
\sum_{p=0}^{\infty} \frac{g^{(p)}(0)}{p !} d_{p} & =\sum_{p=0}^{\infty}(-1)^{p} B_{p} d_{p} \\
& =\sum_{p=0}^{\infty}(-1)^{p} B_{p} \sum_{q=0}^{p}(-1)^{q}\left(\begin{array}{c}
p \\
q
\end{array}\right) c_{q} \\
& =\sum_{q=0}^{\infty} c_{q} \sum_{p=q}^{\infty}(-1)^{p+q}\left(\begin{array}{c}
p \\
q
\end{array}\right) B_{p} \\
& =\sum_{q=0}^{\infty} c_{q} f^{(q)}(0) / q ! .
\end{aligned}
$$

\section{BIBLIOGRAPHY}

1. S. Bernstein, Démonstration du théorème de Weierstrass, fondeé sur le calcul des probabilités, Commun. Soc. Math. Kharkow (2) 13 (1912-13), 1-2.

2. F. Hausdorff, Summationsmethoden und momentfolge I, Math. Z. 9 (1921), 75-109.

3. T. H. Hildebrandt, On the moment problem for a finite interval, Bull. Amer. Math. Soc. 38 (1932), 269-270.

4. G. G. Lorentz, Bernstein polynomials, University of Toronto Press, Toronto, 1953.

5. J. S. Mac Nerney, Characterization of regular Hausdorff moment sequences, proc. Amer. Math. Soc. 15 (1964), 366-368.

6. - Hermitian moment sequences, Trans. Amer. Math Soc. 103 (1962), 45-81.

Received June 15, 1965.

UNIVERSITY OF NORTh CAROLINA 


\section{PACIFIC JOURNAL OF MATHEMATICS}

\section{EDITORS}

H. SAMELSON

Stanford University

Stanford, California

J. P. JANS

University of Washington

Seattle, Washington 98105
J. DugundJI

University of Southern California

Los Angeles, California 90007

RICHARD ARENS

University of California

Los Angeles, California 90024

\section{ASSOCIATE EDITORS}

E. F. BECKENBACH

B. H. NeumanN

F WolF

K. YOSIDA

\section{SUPPORTING INSTITUTIONS}

UNIVERSITY OF BRITISH COLUMBIA

CALIFORNIA INSTITUTE OF TECHNOLOGY

UNIVERSITY OF CALIFORNIA

MONTANA STATE UNIVERSITY

UNIVERSITY OF NEVADA

NEW MEXICO STATE UNIVERSITY

OREGON STATE UNIVERSITY

UNIVERSITY OF OREGON

OSAKA UNIVERSITY

UNIVERSITY OF SOUTHERN CALIFORNIA
STANFORD UNIVERSITY

UNIVERSITY OF TOKYO

UNIVERSITY OF UTAH

WASHINGTON STATE UNIVERSITY

UNIVERSITY OF WASHINGTON

AMERICAN MATHEMATICAL SOCIETY CHEVRON RESEARCH CORPORATION TRW SYSTEMS

NAVAL ORDNANCE TEST STATION

Printed in Japan by International Academic Printing Co., Ltd., Tokyo Japan 


\section{Pacific Journal of Mathematics}

\section{Vol. 21, No. $1 \quad$ November, 1967}

Friedrich-Wilhelm Bauer, Der Hurewicz-Satz................... 1

D. W. Dubois, A note on David Harrison's theory of preprimes . ......... 15

Bert E. Fristedt, Sample function behavior of increasing processes with stationary, independent increments ..................... 21

Minoru Hasegawa, On the convergence of resolvents of operators....... 35

Søren Glud Johansen, The descriptive approach to the derivative of a set function with respect to a $\sigma$-lattice ....................... 49

John Frank Charles Kingman, Completely random measures ............ 59

Tilla Weinstein, Surfaces harmonically immersed in $E^{3} \ldots \ldots \ldots \ldots \ldots . . \ldots 9$

Hikosaburo Komatsu, Fractional powers of operators. II. Interpolation spaces ......................................... 89

Edward Milton Landesman, Hilbert-space methods in elliptic partial differential equations ...................................... 113

O. Carruth McGehee, Certain isomorphisms between quotients of a group algebra ........................................ 133

DeWayne Stanley Nymann, Dedekind groups .................. 153

Sidney Charles Port, Hitting times for transient stable processes ......... 161

Ralph Tyrrell Rockafellar, Duality and stability in extremum problems involving convex functions . ............................ 167

Philip C. Tonne, Power-series and Hausdorff matrices . . .............. 189 